\title{
Parapatric divergence of sympatric morphs in a salamander: incipient speciation on Long Island?
}

\author{
M. CAITLIN FISHER-REID, $\uparrow$ TAG N. ENGSTROM, + CAITLIN A. KUCZYNSKI, \\ PATRICK R. STEPHENS§ and JOHN J. WIENS 9 \\ Department of Ecology and Evolution, Stony Brook University, Stony Brook, NY 11794-5245, USA
}

\begin{abstract}
Speciation is often categorized based on geographic modes (allopatric, parapatric or sympatric). Although it is widely accepted that species can arise in allopatry and then later become sympatrically or parapatrically distributed, patterns in the opposite direction are also theoretically possible (e.g. sympatric lineages or ecotypes becoming parapatric), but such patterns have not been shown at a macrogeographic scale. Here, we analyse genetic, climatic, ecological and morphological data and show that two typically sympatric colour morphs of the salamander Plethodon cinereus (redback and leadback) appear to have become parapatrically distributed on Long Island, New York, with pure-redback populations in the west and pure-leadback populations in the east (and polymorphic populations in between and on the mainland). In addition, the pure-leadback populations in eastern Long Island are genetically, ecologically and morphologically divergent from both mainland and other Long Island populations, suggesting the possibility of incipient speciation. This parapatric separation seems to be related to the different ecological preferences of the two morphs, preferences which are present on the mainland and across Long Island. These results potentially support the idea that spatial segregation of sympatric ecotypes may sometimes play an important part in parapatric speciation.
\end{abstract}

Keywords: amphibians, biogeography, climate, morphology, Plethodontidae, speciation

Received 17 November 2012; revision received 31 May 2013; accepted 3 June 2013

\section{Introduction}

Allopatric speciation is widely considered the most common geographic mode (Coyne \& Orr 2004; Bolnick \& Fitzpatrick 2007). Thus, allopatric and parapatric sister species are often assumed to have originated through allopatric or parapatric speciation, as are species that are partially sympatric (Coyne \& Orr 2004). The possibility that some currently allopatric or parapatric sister species

Correspondence: M. Caitlin Fisher-Reid, Fax: 804-289-8233;

E-mail: cfisherr@richmond.edu

Present addresses: $\uparrow$ Department of Biology, University of Richmond, Richmond, VA 23173, USA

†Department of Biological Sciences, California State University, Chico, CA 95929-0515, USA

§Odum School of Ecology, University of Georgia, Athens, GA 30602, USA

IDepartment of Ecology and Evolution, University of Arizona, Tucson, AZ 85721, USA originated through initial divergence of lineages or ecotypes in sympatry has been suggested (e.g. Coyne 2007; Nosil 2008) and important theoretical studies support this view (e.g. Doebeli \& Dieckmann 2000, 2003), but with few empirical examples (e.g. Seehausen et al. 2008; Ingram 2011). Spatial segregation of sympatric ecotypes along an environmental gradient is a key component of these models and examples. However, the empirical examples involve different habitats at a relatively fine spatial scale (Seehausen et al. 2008; Ingram 2011), and not the macrogeographic scale over which species ranges are usually considered. In some cases, small-scale patterns of spatially segregated ecotypes could be explained by drift or other stochastic factors, whereas large-scale, temporally stable patterns may be more difficult to explain. Here, we address the possibility that sympatric ecotypes within a species can become parapatrically distributed and divergent at a relatively large spatial scale, through a multifaceted analysis of the eastern red-backed salamander 
(Plethodon cinereus) on Long Island, New York (hereafter: 'LI').

Plethodon cinereus ranges from southern Canada to northern North Carolina and typically occurs in two discrete colour morphs: the redback morph and the leadback morph (Petranka 1998). The redback morph (hereafter: 'red') has a broad orange to red dorsal stripe and dark grey flanks, whereas the leadback morph (hereafter: 'lead') is uniformly dark grey on the dorsum and flanks (Petranka 1998). A third, entirely red morph occurs sporadically in some populations (e.g. Tilley et al. 1982). Highton (1975) suggested that the colour morphs are polygenic, with no intermediates, and showed that genetic dominance of morphs varies by population, but there has been no further work on their genetic basis. The function of the red stripe is not entirely clear, but it has been implicated in frequency-dependent selection by predators (Fitzpatrick et al. 2009).
These two morphs are usually found in polymorphic populations with frequencies of approximately $70 \%$ red and $30 \%$ lead, but with red morphs found in much higher proportions (90-100\%) at higher elevations and the northernmost parts of the species range (e.g. Lotter \& Scott 1977; Petranka 1998; Gibbs \& Karraker 2006; Noël \& Lapointe 2010). On LI, however, these two colour morphs are largely parapatric (Fig. 1A), with $100 \%$ red populations in predominantly deciduous western forests, and $100 \%$ lead populations in the eastern pine barrens (Williams et al. 1968). There are also polymorphic populations along the northern shore and in central LI, separating the pure-lead and pure-red populations to the east and west, respectively (Williams et al. 1968).

The red/lead colour polymorphism also occurs in other species of Plethodon. Plethodon contains four wellsupported species groups (e.g. Wiens et al. 2006; Kozak
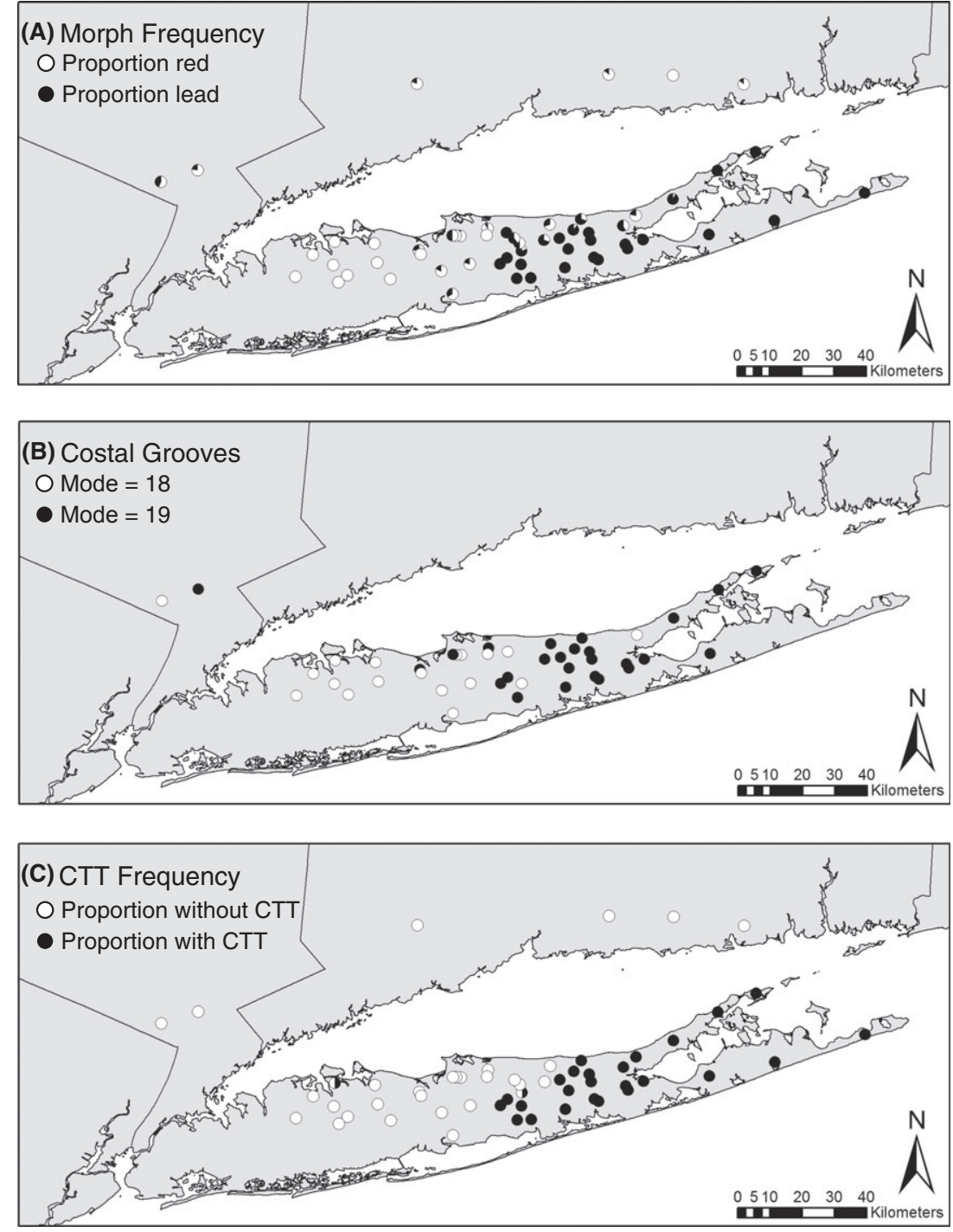

Fig. 1 Maps of LI localities with their (A) morph frequency, (B) modal costal groove number and (C) frequency of CTT mutation in ATPase6. In (A), white is the proportion of red morphs and black the lead morphs. In (B), white represents a modal costal groove count of 18 and black a count of 19. In (C), white is the proportion of individuals that lack the CTT mutation, while black is the proportion having this mutation. For (A, B), only localities with $N \geq 4$ are shown. For (C), all localities have $N=2$. For additional localities with morph frequency and costal groove data, see Figs 1 and 2 in Williams et al. (1968). 
et al. 2009; Fisher-Reid \& Wiens 2011), and eight other species in three of these groups also have sympatric red/lead colour morphs (e.g. P. vehiculum; Petranka 1998). These three groups also contain several apparently monomorphic species (e.g. red: P. idahoensis, lead: P. richmondi; Petranka 1998). These interspecific patterns raise the possibility that fixation of colour morphs or transitions between them might be related to speciation (see below and Discussion).

On the mainland, these morphs differ in ways that may be relevant to speciation. Lead individuals are relatively drought and heat tolerant, whereas red individuals are drought intolerant and cold tolerant (e.g. Williams et al. 1968; Lotter \& Scott 1977; Moreno 1989; Gibbs \& Karraker 2006; Anthony et al. 2008; but see Petruzzi et al. 2006). In polymorphic populations from Ohio, diet differences between colour morphs and moderate assortative mating by colour morph have also been documented (Anthony et al. 2008). Thus, the colour morphs might be relevant to speciation not because of the colours themselves, but rather because different morphs are associated with different environmental tolerances that could lead to occurrence in divergent habitats and subsequently to speciation. However, the cause of the association between colour and climate is currently unknown.

In this study, we test the hypothesis that the currently parapatric colour morphs of P. cinereus on LI evolved from sympatric morphs that have become spatially segregated along an environmental gradient. We also test if these parapatric populations show ecological, genetic and morphological differences suggestive of incipient speciation. From these hypotheses, we make six predictions. First, given a population-level phylogeny, we predict that LI $P$. cinereus populations will form a monophyletic group that is derived from mainland populations where morphs occur in sympatry (i.e. a single population with sympatric morphs invaded LI). Second, we predict that LI populations will cluster as genetically separate from the mainland and that pure-red and pure-lead populations on LI will also form distinct genetic clusters. Third, we expect to find a large-scale environmental gradient that correlates with changes in morph frequency among populations across the island. Fourth, given the ecophysiological differences previously found between sympatric morphs on the mainland (see above), we expect geographic patterns in microclimate occurrence to parallel those for macroclimate. Fifth, we expect that predominantly red and lead populations on LI will diverge in other morphological traits besides colour (e.g. costal groove number; Williams et al. 1968). Sixth, we expect reduced gene flow between populations with divergent morph frequencies, and that, after accounting for geographic distance, gene flow patterns will be related to an environmental gradient.

\section{Materials and methods}

\section{Study system and sampling}

We collected specimens (for morphological data) and tissue samples (for genetic data) from populations of $P$. cinereus across LI $(N=61$ localities) and adjacent mainland areas $(N=21$ localities; for all localities and sample sizes see Table S1, Supporting information). These localities were also used for GIS-based climatic data. A subset of LI, Connecticut and New Jersey localities were used for microclimate measurements. This strategy yielded a matched data set of localities for which we had morphological, genetic, microclimatic and macroclimatic data.

\section{Genetic data}

We analysed patterns of genetic differentiation in $P$. cinereus using both mitochondrial (mtDNA) and microsatellite DNA data. For a complete list of individuals, localities and genetic data collected, see Table S2 (Supporting information).

One mitochondrial gene, ATPase6, was amplified using standard PCR and sequencing protocols (see Table S3 and Text S1, Supporting information for primers, PCR conditions and methods). Variation in ATPase6 was low, and so only 187 individuals from 72 localities (53 LI, 19 mainland) were sequenced. Each locality was represented by 1-6 individuals (mode $=2$ ). Twelve of these $P$. cinereus individuals (seven LI, five mainland) and five additional outgroups were sequenced for cytochrome $b$ (cytb) to assist in rooting the mtDNA tree (see below; Table S2, Supporting information).

Seven microsatellite loci were developed with the cloning protocol outlined in Glenn \& Schable (2005), using a subset of LI P. cinereus (Table S2, Supporting information). We targeted loci variable among LI populations (but not necessarily differing between red and lead populations). Microsatellites identified by Connors \& Cabe (2003) for P. cinereus showed little variation on LI and were not used. Data were obtained for 233 individuals of P. cinereus from 46 localities (31 LI, 15 mainland). Each locality was represented by $2-7$ individuals (mean $=5$ ). See Table S2 (Supporting information) for sampling details, and Table S3 (Supporting information) for microsatellite primers, repeat motifs and PCR conditions. All loci were checked for null alleles, deviation from HardyWeinberg equilibrium, and linkage disequilibrium (see Text S1; Data S1, Supporting information). 


\section{Macroclimate data}

Macroclimate data (Data S2, Supporting information) were obtained for each georeferenced sampling locality using ArcGIS 9.3. Nineteen variables from the WorldClim database were extracted (Hijmans et al. 2005) for 75 localities (58 LI, 17 mainland). These variables summarize temperature and precipitation data from weather stations from 1950 to 2000, spatially interpolated to localities between weather stations, at a resolution of approximately $1 \mathrm{~km}^{2}$.

\section{Morph frequency data}

Morph frequencies (i.e. \% red individuals; Table S1, Supporting information) were estimated for each locality using the same specimens described above. Our estimates are generally concordant with those of Williams et al. (1968). Those authors had larger sample sizes and sampled LI more broadly before much recent urbanization (e.g. they have pure-red localities from southwestern LI). The concordance between our two studies suggests that morph frequencies are largely stable over decadal time scales (but Gibbs \& Karraker 2006 show evidence of small, climate-related frequency shifts). We only used localities with morph frequencies based on at least four individuals (range: 4-25; mean: 11).

\section{Microclimate data}

Fine-scale environmental data (microclimate data hereafter) included soil moisture (\%), soil $\mathrm{pH}$, ground temperature $\left({ }^{\circ} \mathrm{C}\right)$, air temperature $\left({ }^{\circ} \mathrm{C}\right)$ and air humidity ( $\%$ relative humidity). Data were collected weekly from randomly selected localities, between March and November during 2009-2011 (Data S3, Supporting information). Each visit began between 13:00-15:00 hours and lasted 1-2 hours. Five, nonoverlapping 20-m transects were conducted sequentially in forested areas with a high density of cover objects (e.g. logs, rocks). Cover objects within $2 \mathrm{~m}$ of the transect tape were searched for salamanders. When a salamander was found, its colour morph and microclimate data were recorded. See Text S1 (Supporting information) for details on field sampling methods (e.g. locality selection, sampling frequency).

\section{Morphometric data}

Morphometric data were collected from 414 preserved specimens from LI and the mainland (Data S4, Supporting information). Measured individuals had a total length (snout-vent-length + tail length) $\geq 60 \mathrm{~mm}$, to exclude juveniles (adult total length approximately 65-125 mm;
Petranka 1998). Nine measurements were recorded for each specimen (see Text S1, Supporting information).

\section{Mitochondrial data analyses}

A likelihood analysis of the combined mtDNA data (ATPase6, cytb) was conducted using RAxML ver. 7.2.6 (Stamatakis 2006). Methodological details are described in Text S2 (Supporting information). The mtDNA analysis contained several outgroup species from the cinereus group, and Ensatina eschscholtzii to root relationships among $P$. cinereus populations and help identify a root for the microsatellite tree (given the difficulty of amplifying microsatellites across species). In the mtDNA tree (Fig. S1, Supporting information), a clade of predominantly southeastern populations is sister to all other $P$. cinereus populations. Therefore, the microsatellite tree was rooted with Virginia populations, a decision further supported by the fact that the putative sister species to P. cinereus, P. shenandoah (Kozak et al. 2009; Fisher-Reid \& Wiens 2011; this study) is also endemic to Virginia (Petranka 1998). However, rooting should have little impact on our conclusions, as long as the root is not within LI.

\section{Microsatellite data analyses}

First, to address monophyly of LI P. cinereus, a FitchMargoliash tree was constructed using Cavalli-Sforza \& Edwards (1967) chord distances $\left(D_{C}\right)$ in Phylip ver. 3.69 (Felsenstein 2004). Populations (i.e. localities) were the terminal units, with data from two to seven individuals per locality (mean $=5$ ). Takezaki \& Nei (1996) showed that $D_{C}$ was the best of seven distances when dealing with highly variable markers like microsatellites. Clade support was evaluated using 1000 bootstrap replicates. Two Virginia localities of $P$. cinereus were used as outgroups (see above).

In order to visualize the geographic origin of populations (LI vs. mainland) and the general morph frequency category of populations (polymorphic, monomorphic red, monomorphic lead), we reconstructed geographic origin and morph category as two separate characters on the microsatellite tree using maximum-likelihood in Mesquite ver. 2.73 (Maddison \& Maddison 2011). We used the Mk two-rate model for geographic origin and Mk onerate model for morph frequency category (the latter is the most straightforward model for three-state characters). We acknowledge that character reconstruction methods are designed for species and not populations (and not for geographic origins), but these analyses were used to visualize the general patterns implied by the microsatellite data. Additionally, to reduce biases from placing populations in discrete categories (i.e. polymorphic, fixed), we 
also conducted reconstructions treating locality morph frequencies as a continuous trait (see Text S2, Supporting information).

We conducted a Bayesian individual-based clustering analysis of the microsatellite data using STRUCTURE ver. 2.3.3 (Pritchard et al. 2000; Falush et al. 2003). This provided an additional test of the genetic distinctness of the mainland and LI populations, and red and lead populations on LI. We used location priors, correlated allele frequencies and a 'no admixture' model of ancestry for the following reasons. First, our data are composed of relatively few individuals per locality (mode $=5$ ) and markers (seven microsatellite loci), and the use of location priors can assist STRUCTURE in clustering without biasing analyses towards detecting spurious structure (Hubisz et al. 2009; Pritchard et al. 2010). Second, we expect LI cinereus populations to share some alleles due to common ancestry (in which case correlated allele frequencies should be used; Falush et al. 2003), given their very large population sizes (Petranka 1998) and an mtDNA tree with little geographic substructuring (Fig. S1A, Supporting information). Finally, the 'no admixture' model was selected given that most $P$. cinereus on LI currently occur in human-caused forest fragments, and are thus currently isolated from each other (i.e. not likely to be experiencing current genetic admixture; e.g. Jordan et al. 2009).

The number of distinct populations (K) supported was estimated with STRUCTURE by comparing likelihood scores using values of $K$ from 1 to 15 . Each $K$ was run for five replicates using a burn-in of 100000 steps, followed by $2 \times 10^{6}$ steps. Results were analysed using Structure Harvester web version 0.6.92 (Earl \& vonHoldt 2011), which extracts the mean and standard deviation of $\log$ likelihood $[\operatorname{Ln} \mathrm{P}(K)]$ for each $K$, as well as the $\Delta K$ metric of Evanno et al. (2005; the rate of change of the likelihood function). Both mean Ln $\mathrm{P}(K)$ and $\Delta K$ were used to determine the best $K$ (Evanno et al. 2005; Pritchard et al. 2010).

\section{Macroclimatic data analyses}

In order to test for a macroclimatic gradient on LI, we performed a principal component analysis (PCA) on the correlation matrix of the 19 climatic variables (Table S4, Supporting information) using JMP ver. 9.0.2. PC 1 and 2 , which together account for $64.69 \%$ of the variation in climate, were plotted to visualize major climatic differences between localities. PC 1-3 each accounted for more variation than expected by chance (broken stick; Jackson 1993). These PC scores and select raw variables (BIO1, annual mean temperature; BIO12, annual precipitation) were tested for correlation with locality morph frequencies using Spearman's rank correlation in $\mathrm{R}$, given the non-normality of the variables (Shapiro-Wilk test).

\section{Microclimatic data analyses}

We tested for differences in microclimate between four types of localities: (i) LI pure-red, (ii) LI pure-lead, (iii) LI polymorphic and (iv) mainland. Field data for 26 localities (22 LI, four mainland) and 493 salamanders (344 red, 149 lead) were pooled first by locality (all salamanders at a given locality across time), and then by the four groups listed above. While not ideal (given temporal variation), sampling was too limited across all time scales at a given locality to remove temporal effects (Data S3, Supporting information). Means of microclimate variables were estimated for each group. Importantly, these data represent microclimate for individual salamanders. Therefore, microclimate data can reflect individual preferences, because salamanders actively select their microhabitat (Petranka 1998). Each microclimatic variable was included in the following statistical analyses, conducted in R: (i) LI pure-red vs. LI pure-lead (Wilcoxon rank sum two-sample test); (ii) LI pure-red vs. LI polymorphic vs. LI pure-lead (Kruskal-Wallis test); and (iii) LI pure-red vs. LI polymorphic vs. LI pure-lead vs. mainland (Kruskal-Wallis test). Again, given the non-normality of the data (ShapiroWilk test), we used Spearman's rank correlation to estimate the relationship between-locality morph frequency and the locality mean for each microclimate variable.

\section{Morphological data analyses}

We tested for differences between populations and colour morphs by performing two PCAs on the correlation matrix of the morphological data. The first PCA included costal groove number plus eight morphological variables (Text S1, Supporting information), whereas the second excluded costal groove number. Costal grooves are external indentations in the thoracic wall that generally reflect vertebral number (Petranka 1998). Costal groove number is problematic, as it exhibits a limited range of values among these populations and is therefore not truly continuous. In both PCAs, PC1 was the only PC to account for more variation than expected by chance (broken stick; Jackson 1993). However, as is typical for morphology, PC1 appears to primarily reflect size (i.e. loadings positive and similar in magnitude). Thus, we included PC2, as a measure of shape. Mean PC scores were estimated for each locality, and we tested for a relationship between morphology (PC1 and PC2, with and without costal grooves), and colour morph frequency with Spearman's rank correlation (given non-normality; Shapiro-Wilk test). 


\section{Spatial analyses}

We compared pairwise $F_{\mathrm{ST}}$ values in order to test whether or not LI populations were more genetically divergent (i.e. had higher $F_{\mathrm{ST}}$ values) than mainland populations over similar geographic distances. Initially, we looked at a subset of mainland localities and the centres of each group of LI localities (LI pure-red, LI pure-lead, LI polymorphic). The LI groups were compared to seven nearby mainland localities and arranged roughly in a west-east line (i.e. in Connecticut, New York and New Jersey; six are pictured in Fig. 1A). We estimated pairwise $F_{\mathrm{ST}}$ (Weir \& Cockerham 1984) values using the microsatellite data and GENEPOP on the Web (Raymond \& Rousset 1995; Rousset 2008). For this analysis, we estimated the geographic midpoint location of LI pure-red, LI polymorphic and LI pure-lead locality groups by averaging the latitude and longitude for each group (Fig. S2, Supporting information). These midpoints were used to estimate the geographic distance in $\mathrm{km}$. Next, we estimated pairwise $F_{\mathrm{ST}}$ values and geographic distance between all single localities and used these values as a distance measure in a partial Mantel test with a group membership matrix (LI pure-red, LI pure-lead, LI polymorphic, mainland). This analysis tests the hypothesis that differences in group membership predict differences in pairwise $F_{\mathrm{ST}}$ when accounting for geographic distance (i.e. individuals from different localities in the same group have lower $F_{\mathrm{ST}}$ than those in different groups; see below). This analysis was also repeated with LI groups only.

Finally, we conducted a series of single and partial Mantel tests (Mantel 1967; Smouse et al. 1986; Legendre \& Legendre 1998) to examine if our predictor variables (macroclimate, microclimate) predict variation in our response variables (genetic distance, locality morph frequency) while accounting for spatial autocorrelation. We acknowledge that there have been important criticisms of these widely used tests (e.g. Guillot \& Rousset 2013), but we use them here because well-established alternative methods are not yet available. Fortunately, the significant patterns identified by these tests generally match those found in our corresponding non-Mantel analyses. Additionally, given the ability of $P$. cinereus to rapidly disperse over very large geographic areas (e.g. postglacial colonization of Canada), it is unlikely that the patterns we find are explained by limits on dispersal due to geographic distance alone.

These analyses were conducted both with LI and mainland populations, and with LI populations alone. Specifically, we tested the following five hypotheses (sample sizes in Tables S9a-b, Supporting information), while accounting for geographic distance between localities: (i) differences in macroclimate between localities predict differences in morph frequency; (ii) macroclimate predicts genetic distance; (iii) microclimate predicts morph frequency; (iv) microclimate predicts genetic distance; and (v) macroclimate predicts microclimate. Because macroclimate and microclimate include several variables of differing scales (e.g. temperature, precipitation), each group of variables was first put through a PCA of the correlation matrix, and a Euclidean distance matrix (Text S2, Supporting information) was then estimated from the PC scores (19 for macroclimate, five for microclimate). Separate PCAs were conducted for combined LI-mainland data (Table S4a, Supporting information) and the LI data only (Table S4b, Supporting information). Text S2 (Supporting information) contains a description of distance matrices and their estimation.

All Mantel tests were conducted using PASSaGE ver. 2.0 (Rosenberg \& Anderson 2011), with significance determined using 10000 permutations. Because each hypothesis requires six statistical tests (three each, single and partial Mantel tests), a sequential Bonferroni correction (Rice 1989) was applied for each hypothesis.

\section{Results}

\section{Microsatellite data analyses}

Phylogenetic analysis of the microsatellite data (Fig. 2) shows that LI populations form a monophyletic group nested within mainland populations, although the bootstrap support is low. Likelihood reconstructions on the microsatellite tree suggest that the ancestor of all LI $P$. cinereus populations was polymorphic (i.e. sympatric morphs) and that there was subsequent evolution of pure-lead populations on eastern LI and pure-red populations on western LI (Fig. 2; Fig. S3, Supporting information). Pure-lead populations appear to have evolved once on LI (the clade of eastern LI populations) with possible introgression with adjacent polymorphic populations on the eastern north shore (Cutchogue, Northville). In contrast, pure-red populations appear to have evolved approximately five times on western LI (Fig. 2). The designation of some of these populations as pure-red may reflect limited sampling (e.g. Brentwood Highway 4; $N=4$ ). However, a group of pure-red populations near Oyster Bay (Old Westbury, Oyster Bay, Woodbury) is supported by very large sample sizes (hundreds of specimens; Williams et al. 1968; recent field visits).

In the STRUCTURE analyses of the microsatellite data, $\Delta K$ favours $K=2$, whereas log likelihood favours $K=9$ (Table S5, Supporting information). The results for $K=2$ (Fig. 3 ) divide $P$. cinereus into one exclusively LI cluster and a second cluster consisting of all mainland populations and a few LI populations 


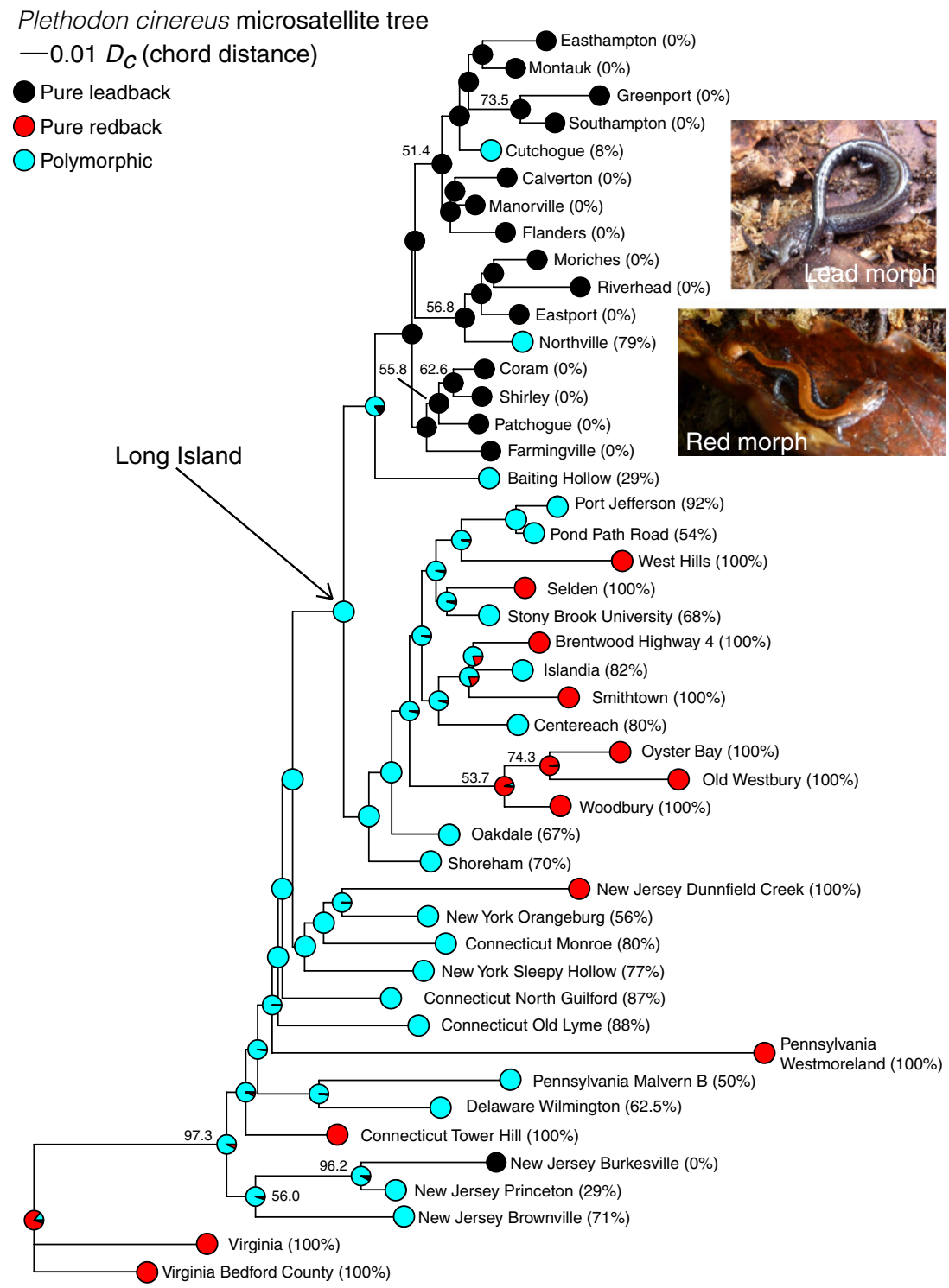

Fig. 2 Tree of Plethodon cinereus populations based on microsatellite data. Bootstrap values $<50$ are not shown. Pie charts at nodes show likelihood ancestral reconstruction of morph categories. The morph frequency of the locality is in parentheses following its name. Photographs by M.C.F.R.

(all pure-red). In the $K=9$ results (Fig. 3), there are five mainland clusters and four LI clusters. The LI clusters correspond to a pure-red cluster, a pure-red/ polymorphic cluster, a pure-lead/polymorphic cluster and a pure-lead cluster. These clusters broadly match clades recovered in the phylogenetic analysis (Fig. 2). Interestingly, $K=9$ is the next best model favoured by $\Delta K$ (Table S5, Supporting information). Generally, $\Delta K$ is thought to capture the strongest pattern of population structure (e.g. in our case, most LI vs. the mainland; Evanno et al. 2005; Coulon et al. 2008), and both likelihood and the second best $\Delta K$ reflect the structure on LI. Overall, the STRUCTURE results largely support the genetic distinctness of the mainland and LI populations ( $K=2$ shows strong divergence between mainland and most LI populations; $K=9$ shows effectively no overlap between LI and mainland). Although results from $K=2$ might be interpreted as indicating recent gene flow between mainland and pure-red populations, this is not supported by phylogenetic analysis or more fine-scaled analysis with STRUCTURE $(K=9)$.

\section{Mitochondrial data analyses}

Phylogenetic analysis of the mtDNA data (Fig. S1, Supporting information) included 101 unique ATPase6 haplotypes. Plethodon shenandoah is sister to all P. cinereus individuals (consistent with Wiens et al. 2006; Kozak et al. 2009; Fisher-Reid \& Wiens 2011), and a clade of mainland $P$. cinereus individuals from mostly southern populations (Virginia, Delaware, Maryland and North Carolina, but also New York) is sister to all other $P$. cinereus, including all LI populations. Interestingly, 24 individuals from pure-lead localities on LI form a 

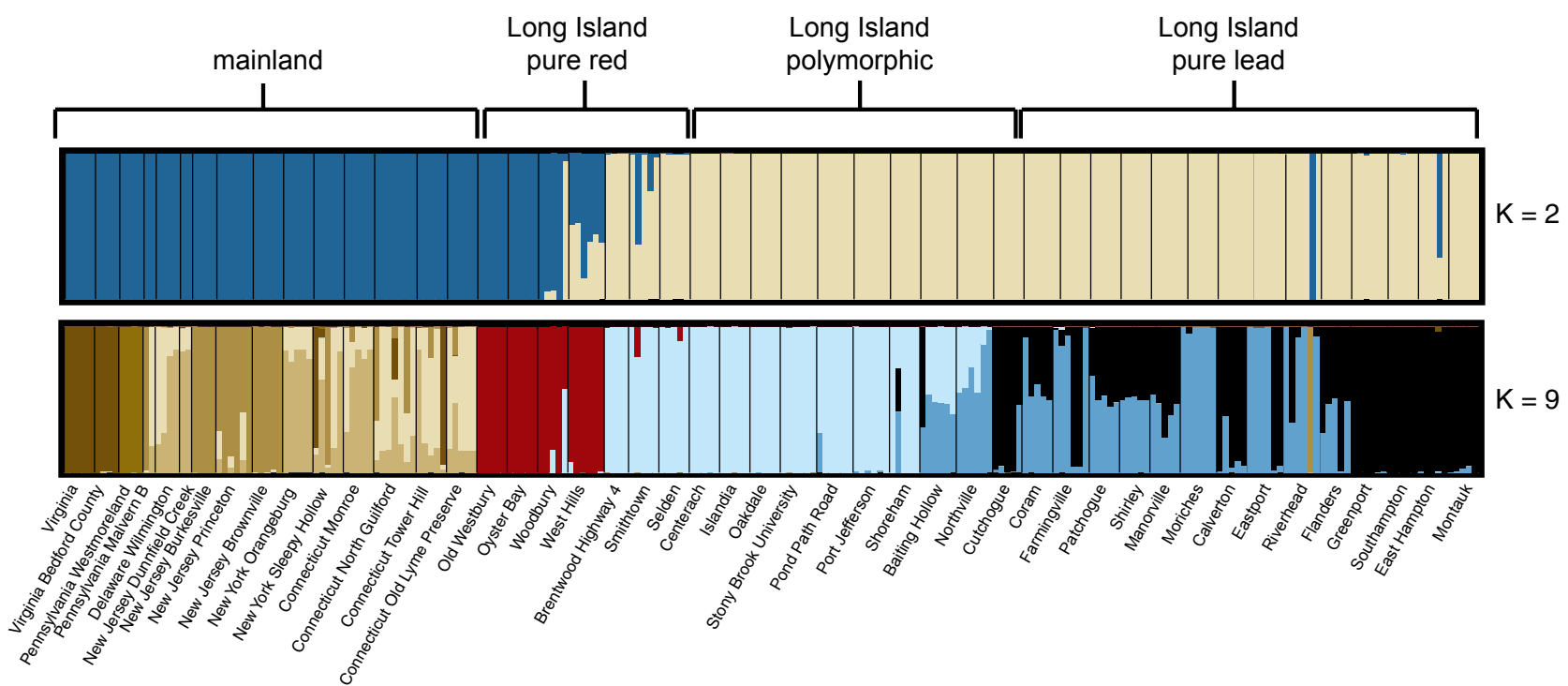

Fig. 3 Results from Bayesian cluster analysis of the microsatellite data (using STRUCTURE), showing bar plots for the optimal $K$ values of 2 and 9. Each single line is an individual, and each group of lines (denoted by black separator bars) is a group of individuals from the same locality ( $N=46$ localities). Each genetic cluster is represented by a unique colour, and the individuals are colour ed to represent their estimated membership fraction for each genetic cluster. For $K=2$ (best $\Delta K$ ), there is a pure LI cluster of mostly polymorphic and pure-lead populations, and a mainland/LI cluster containing mainland and some pure-red populations. For $K=9$ (best log likelihood score), there are five mainland clusters and four LI clusters. These LI clusters separate partially by population morph frequency, although there is overlap between pure-reds and polymorphic, and pure-leads and polymorphic. Note that a single Riverhead individual clusters with mainland groups in both plots. We hypothesize that this individual was transplanted to LI recently by humans.

weakly supported clade separate from the rest of the LI individuals. These individuals share a single, nonsynonymous nucleotide change in ATPase6 (from TTT to CTT; Fig. 1C). Two additional individuals in this clade are red morphs, one from a polymorphic locality on the northeastern shore (Wading River) and one from a pure-red locality (Huntington). No mainland populations exhibit this mutation.

\section{Macroclimate data analyses}

In the PCA of macroclimatic variables (Fig. 4), PC1 separates mainland from LI localities, whereas PC2 separates the LI localities along a climatic gradient that roughly corresponds to morph frequencies. Across all localities, locality morph frequency was strongly correlated with PC1-3 (PC1: $r_{s}=0.588, P \ll 0.0001 ;$ PC2: $r_{s}=0.454, P=0.0002 ;$ PC3: $\left.r_{s}=0.503, P<0.0001\right)$, but not with raw values of annual mean temperature (BIO1) or annual precipitation (BIO12). The variables that load strongly onto PC1 (BIO6, BIO11, BIO19; Table S4, Supporting information) suggest that LI has warmer and wetter winters than the mainland, whereas the variables that load strongly onto PC2 (BIO1, BIO5, BIO10, BIO12, BIO19; Table S4, Supporting information) suggest that pure-lead populations experience wetter winters, and overall cooler temperatures compared to pure-red populations. PC3 is dominated by strong loadings from precipitation variables (BIO12, BIO14, BIO15, BIO17; Table S4, Supporting information) and suggests that most pure-lead populations experience a drier, more seasonal precipitation regime.

\section{Microclimate data analyses}

Statistical analyses of the microclimate data (Table S6, Supporting information) show that individuals from pure-lead localities on LI are active during significantly colder, drier conditions and in drier, more basic soil than those found at pure-red localities $(W=1927.5$ 4012.0; $P \leq 0.00384$ for soil moisture, $\mathrm{pH}$, ground and air temperatures). Interestingly, the temperature pattern is opposite that observed in previous mainland studies, which indicated that leads preferred warmer temperatures (and lower elevations; see Introduction), while the moisture pattern on LI is consistent with mainland studies. As an island, coastal insulation leads to warmer overall temperatures on LI; thus, the colder temperatures that pure-leads experience are still warmer than those encountered by both morphs on the mainland (Table S6, Supporting information). Both microclimatic patterns correspond with the macroclimatic patterns 


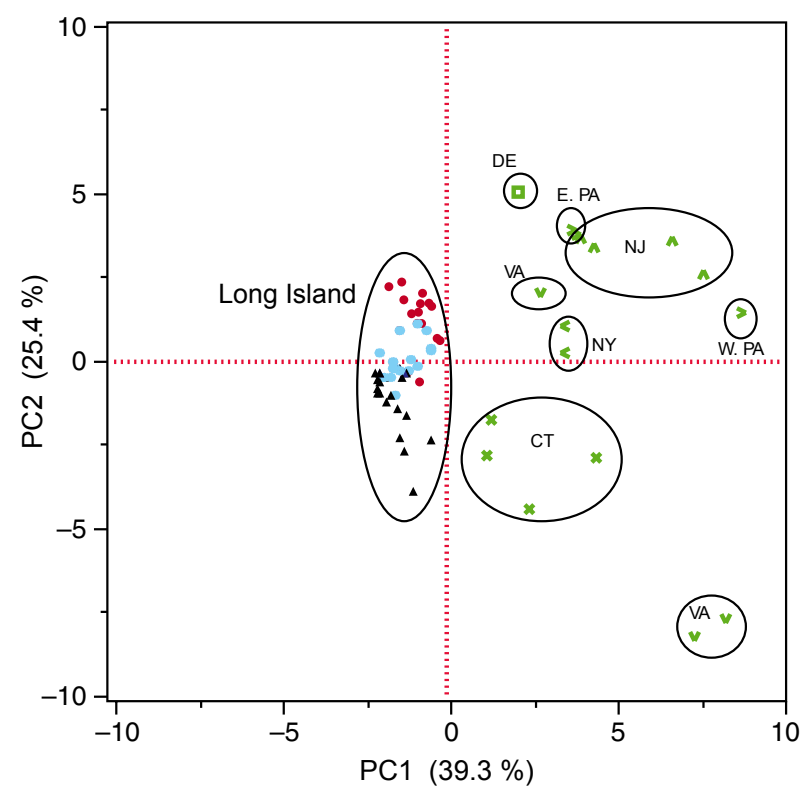

Fig. 4 Localities of Plethodon cinereus plotted in multivariate climatic niche space (PC1 vs. PC2; see Table S4a, Supporting information for loadings). Green symbols are mainland localities, with different states indicated with different shapes. Red circles are pure-red LI localities, light blue circles are polymorphic LI localities and black triangles are pure-lead LI localities.

(i.e. higher lead frequencies in cooler/drier localities). Microclimatic differences are nonsignificant when comparing all red individuals on LI to all lead individuals on LI (i.e. including reds and leads from polymorphic populations; Table S6, Supporting information). At LI polymorphic localities, lead morph salamanders were encountered on significantly warmer and less humid days compared to red morph salamanders (air temperature: $W=2736.5 ; \quad P=0.032$; air humidity: $W=3640$; $P=0.021$ ), as found in previous mainland studies (see Introduction). A Kruskal-Wallis test comparing purered, pure-lead and polymorphic localities found polymorphic localities to be significantly different from and intermediate between pure-red and pure-lead localities for soil moisture and ground temperature (soil moisture: $\chi^{2}=8.17 ; \quad P=0.017 ;$ ground temperature: $\chi^{2}=8.85$; $P=0.012$ ). LI salamanders are active under significantly warmer microclimatic conditions than mainland individuals (Kruskal-Wallis, $\chi^{2}=89.32-97.27 ; P<<0.0001$ ), confirming the macroclimatic pattern. The absence of salamanders at both mainland and LI localities appears to be related to ground and air temperatures: days on which no salamanders were found were significantly warmer than those on which salamanders were found $(W=5505-9725$; all $P<0.006)$. Despite these differences among groups of localities (i.e. pure-red, pure-lead, polymorphic), Spearman's rank correlation of the mean microclimatic data (five raw variables) and locality morph frequency for each individual locality revealed no significant relationships between microclimatic variables and morph frequency (all $P>0.1$ ).

\section{Morphological data analyses}

Costal grooves show a pattern of variation that closely matches the morph frequencies (Fig. 1A,B). In pure-lead populations, the modal number of costal grooves is larger (19 vs. 18), as shown by Williams et al. (1968). A PCA of morphology including costal groove number revealed a separation between reds and leads on LI along PC2 (Table S7; Fig. S4, Supporting information). Additionally, PC2 including costal groove count correlated strongly with locality morph frequency $\left(r_{s}=-0.807\right.$, $P \ll 0.0001)$. In contrast, there was no correlation between morphology and locality morph frequency on PC1 including costal grooves or on PC1-2 not including costal grooves (all $P>0.4$ ).

\section{Spatial analyses}

The $F_{\mathrm{ST}}$ between LI pure-red and LI pure-lead localities is higher than between any of the mainland localities along a similar west-east gradient, including the two most distant from each other (New Jersey-Dunnfield Creek and Connecticut-Old Lyme; Table S8, Supporting information). For example, the centres of LI pure-red and LI pure-lead localities are separated by $78.63 \mathrm{~km}$ and have an $F_{\mathrm{ST}}$ of 0.217 . Mainland locality pairs Connecticut-Tower Hill/Connecticut-Monroe, and New York-Orangeburg/Connecticut-Monroe are separated by 79.51 and $79.92 \mathrm{~km}$, and have an $F_{\mathrm{ST}}$ of 0.069 and 0.097 (respectively; Table S8, Supporting information). A partial Mantel test of the pairwise $F_{\mathrm{ST}}$ matrix for all localities against the predictor matrix of group membership reveals a significant correlation between $F_{\mathrm{ST}}$ and group membership when geographic distance is accounted for (including mainland: $r=0.424, P=0.0001$, Table S9a; LI only: $r=0.406, P=0.0001$, Table S9b, Supporting information).

The single and partial Mantel tests for the combined LI-mainland data (Table S9a, Supporting information) show that macroclimatic distance has a significant relationship with both genetic distance and locality morph frequency, even when the strong correlation between macroclimate and geographic distance is accounted for. All relationships hold when considering LI data alone (Table S9b, Supporting information). Finally, for both data sets, microclimatic distance does not predict either genetic distance or locality morph frequency, nor is it related to macroclimatic or geographic distance. 


\section{Discussion}

In this study, we tested the hypothesis that divergent parapatrically distributed populations can arise through geographic segregation of formerly sympatric ecotypes along a large-scale environmental gradient. Through a multifaceted analysis of $P$. cinereus salamanders, we show that ecologically differentiated colour morphs that are sympatric on the mainland appear to have become parapatrically distributed on LI and that these parapatric populations have further diverged genetically, ecologically and morphologically. We suggest that the divergence of pure-lead P. cinereus on eastern LI may represent incipient speciation (although they are clearly not separate species yet). Many other parapatrically distributed sets of populations and species may also arise through geographic separation of sympatric ecotypes along an environmental gradient. However, such cases may easily be overlooked if the ecological and morphological differences between these ecotypes are subtle (i.e. in our study, the ecotypes have clear differences in colour, but different ecotypes within a species or population might often lack such obvious morphological markers).

We acknowledge that the scenario of parapatric differentiation on LI is difficult to prove conclusively. Nevertheless, this scenario is supported both by our genetic data and by other lines of evidence. Under this scenario, $P$. cinereus on LI are derived from an ancestrally polymorphic mainland population. Phylogenetic analysis of our microsatellite data suggests that LI P. cinereus are derived from a polymorphic mainland population (Fig. 2), while STRUCTURE analysis of these data shows that LI $P$. cinereus are largely distinct from mainland populations ( $K=9$; Fig. 3). Thus, the microsatellite results also support the idea that LI populations diverged genetically after colonizing LI (Figs 2 and 3).

The scenario of parapatric segregation of sympatric ecotypes is also supported by the well-known geological history of LI. Long Island was formed by the receding Wisconsin ice sheet relatively recently $(25-30 \mathrm{kyr}$ ago) and was likely only accessible to these salamanders for a brief period in the last 10-15 kyr (Williams et al. 1968). Thus, P. cinereus on LI must have arrived recently from the mainland, and they are currently surrounded by polymorphic mainland populations (Fig. 1; Williams et al. 1968). As the glacier re-treated and sea levels began to rise, $P$. cinereus is thought to have rapidly expanded its range from southern refugia (Bloom 1983; Petranka 1998). Individuals from polymorphic southern populations on the exposed continental shelf were likely forced to move north by the encroaching Atlantic Ocean and became trapped on LI (Bloom 1983; Lewis \& Stone 1991; Stone et al. 2005). Importantly, sea level has never been higher than it is now (Bloom \& Stuiver 1963); thus, the differentiation of populations seen now most likely reflects responses to the current environmental gradient, rather than allopatric differentiation of populations on smaller islands.

The hypothesis of parapatric divergence is further supported by evidence of an environmental gradient across LI, along which populations have diverged genetically and morphologically (Fig. 4; Table S9b, Supporting information). Such gradients are key components of previous models (Doebeli \& Dieckmann 2000, 2003; Gavrilets 2004) and empirical studies (Seehausen et al. 2008; Ingram 2011) of speciation via geographic segregation of formerly sympatric ecotypes. Based on PC2 and PC3, LI pure-lead populations occur in overall colder and drier environments that also have warmer and wetter winters. The overall temperature gradient is striking in that mainland leads appear to prefer warmer temperatures (e.g. Lotter \& Scott 1977; Gibbs \& Karraker 2006; Anthony et al. 2008). Petruzzi et al. (2006) found divergent temperature tolerances (as measured by maintenance metabolic rates) between morphs at different localities, which suggests that temperature tolerance may simply be more variable than moisture tolerances. However, within polymorphic populations on LI, we confirm that leads are active on warmer, less humid days than reds (as in mainland populations), and we note that pure-lead localities on LI have overall warmer microclimates than mainland localities (Table S6, Supporting information). The between-locality microclimatic data largely confirm the macroclimatic patterns, suggesting that morph-specific physiological tolerances are likely. Interestingly, our results suggest that LI has a unique climate and a unique climatic gradient (Fig. 4), which may help explain why the pattern of pure-red and pure-lead populations in close proximity does not occur on the mainland (but pure-red populations do occur in cooler areas).

Remarkably, the pattern on LI appears to be geographically replicated on the Delmarva peninsula (Delaware-Maryland-Virginia; Highton 1977) and may have similar ecological causes. Sampling by Highton (1977) over a 20-year period yielded 177 localities with thoroughly estimated morph frequencies. As on LI, localities closer to the mainland are pure-red, whereas those farthest from the mainland (southern end) are pure-lead, with polymorphic populations in between. We extracted climatic data for 176 of Highton's (1977) localities (excluding one imprecise locality; Data S5, Supporting information) and tested for a correlation between morph frequency and raw climatic variables, focusing on climatic variables (defined in Table S4a, Supporting information) loading strongly onto PC2 and PC3 (which separate pure-red and lead LI populations; Table S4a, 
Supporting information). We found significant negative correlations between the proportion of red individuals and $\mathrm{BIO} 1$ and $\mathrm{BIO} 10$ (which load strongly on PC2; higher red frequency in colder climates; BIO1: $r_{s}=-0.177$, $P=0.019$; BIO10: $\left.r_{s}=-0.229, P=0.002\right)$, and significant positive correlations between red frequency and BIO12, BIO14 and BIO17 (loading strongly on PC3; higher lead frequency in drier climates; BIO12: $r_{s}=0.314, P \ll 0.001$; BIO14: $\quad r_{s}=0.379, \quad P \ll 0.001 ; \quad$ BIO17: $\quad r_{s}=0.276$, $P<0.001)$. Overall, the concordance of these results from the Delmarva peninsula with those from LI further suggests that the patterns on LI are related to ecological segregation due to climate-related factors and not chance, genetic drift, double colonization or other scenarios.

We suggest that LI P. cinereus might represent incipient speciation, especially the pure-lead populations on eastern LI. Three lines of evidence support this hypothesis: restricted gene flow, local adaptation and parallels between the within-species pattern in P. cinereus and between-species patterns in other Plethodon. First, as described above, pure-lead populations on eastern LI are more genetically different from other LI populations than pairs of mainland localities that are far more geographically distant (Table S9, Supporting information). The genetic distinctness of pure-lead eastern LI populations is also supported by the phylogenetic analysis of the microsatellite data (Fig. 2). However, even in these pure-lead populations, there is evidence for introgression with far eastern polymorphic LI populations (e.g. Figs 2 and 3). Clearly, LI P. cinereus are not two distinct species yet, although there is evidence for restricted gene flow between pure-lead and other populations (Table S9, Supporting information).

A second line of evidence for incipient speciation on LI is that some data suggest local adaptation to different environments, which could lead to further genetic divergence. Individuals from pure-lead populations tend to have more costal grooves (Fig. 1B; see also Williams et al. 1968). Costal groove number correlates with vertebral number, which is known to vary in plethodontids and can have a strong genetic component (Jockusch 1997). Jockusch (1997) and others (e.g. Wake 1966) hypothesized that increased vertebral number is an adaptation for a fossorial lifestyle. Eastern LI (where pure-lead populations occur) is dominated by pine barrens with loose, sandy soil and individuals there may benefit from being better adapted to burrowing (Williams et al. 1968). Although we found little evidence for divergence in other morphometric variables, plethodontids generally show remarkably similar body shapes across divergent microhabitats (Blankers et al. 2012). Additionally, individuals from pure-lead populations share a nonsynonymous change in ATPase6 (Fig. 1C), which (along with other changes) might be related to metabolic differences between morphs and populations (given metabolic differences previously documented between morphs; Moreno 1989). The adaptive significance of both traits will need to be investigated in future studies.

The third line of evidence for incipient speciation is the parallel between within-species patterns in LI $P$. cinereus and patterns of between-species divergence among other Plethodon. Several other Plethodon species are polymorphic for red/lead colour morphs, and several others appear to be fixed for one morph or the other (Petranka 1998; Anthony et al. 2008). This suggests that the colour polymorphism may be associated with speciation, although it seems unlikely to be driving speciation itself (see Fitzpatrick et al. 2009 for discussion of colour polymorphism function). Based on this study and others (e.g. Lotter \& Scott 1977; Gibbs \& Karraker 2006; Anthony et al. 2008), colour does appear to be linked to climatic distributions in P. cinereus, and some assortative mating by colour has been found (Anthony et al. 2008). Divergent climatic distributions can drive geographic isolation, adaptation and genetic divergence between populations (as found here), especially if individuals of one incipient species cannot tolerate the climatic conditions experienced by the other, and many authors have suggested that this general process can lead to speciation (e.g. Moritz et al. 2000; Kozak \& Wiens 2007; Sobel et al. 2010). Across plethodontids, rates of species diversification (speciation-extinction) are higher in clades with faster rates of climatic niche evolution (Kozak \& Wiens 2010). Interestingly, our preliminary results examining realized climatic niches of polymorphic and monomorphic Plethodon species climatic data from Kozak \& Wiens 2010; Fisher-Reid et al. 2012 suggest that between-species patterns of climatic distributions in monomorphic species do parallel the within-species patterns observed in $P$. cinereus, with pure-red species in cooler, wetter environments (MC Fisher-Reid \& JJ Wiens, unpublished). We emphasize that we do not consider the colour morphs themselves to be likely drivers of speciation, but rather we hypothesize that these colour morphs are somehow associated with different environmental preferences and tolerances that are then determined by less obvious traits (such as physiology and behaviour).

We recognize that much additional work is needed on this system. First, as discussed above, the relationship between colour morphs, climate and speciation could be examined across species. The physiological tolerances of both $P$. cinereus morphs and those of other species should also be studied in more detail (e.g. Moreno 1989; Petruzzi et al. 2006). Second, analysis of LI P. cinereus using data from next-generation sequencing could provide clues to the causes of the apparent association 
between colour and climate (e.g. detecting loci under selection, detecting associations of particular alleles with particular habitats), and yield stronger support for the relationships and origins of LI cinereus populations. Third, behavioural tests could provide insights into the evolution of prezygotic reproductive isolation. More work is also needed to determine whether the existing patterns of morph distribution and genetic divergence are directly related to different climatic regimes, or possibly to other factors that might be themselves caused by differences in climate (e.g. occurrence in deciduous forests vs. pine barrens, differences in predation). Finally, if there is a speciation-driving environmental gradient on LI, we might expect to find similar, though perhaps morphologically cryptic, patterns in other species distributed across the island (Coyne \& Orr 2004).

In conclusion, we have presented evidence for parapatric segregation of formerly sympatric morphs at a large geographic scale in association with an environmental gradient. Pure morph populations are divergent ecologically, morphologically and genetically, suggesting that this process might eventually lead to parapatric speciation. This pattern of parapatric divergence of formerly sympatric ecotypes along an environmental gradient may represent an early part of the process of parapatric speciation (or range expansion) in many other systems, but may be much harder to detect because the different ecotypes are not as obvious as colour morphs in $P$. cinereus. This pattern of spatial segregation of pre-existing ecotypes might also allow parapatric speciation to happen more quickly, as the presence of sympatric ecotypes with divergent tolerances may reduce the need to wait for new mutations to allow invasion of a new environment.

\section{Acknowledgements}

We thank T. C. Glenn for assistance in developing microsatellite loci. We thank E. Callender, A. Dunham, V. Rudolf and C. Wolfe for help with initial fieldwork. We thank C. Wolfe for help with initial aspects of the project, and S. Smith and C. Ulloa for help with preliminary microclimatic data. We thank many Stony Brook University undergraduates who helped collect microclimate data: R. Ahmed, R. Bacchus, T. Bonadonna, W. Chan, P. Davis, A. Golwala, C. Hiu, J. Hubbard, T. Leong, K. Leung, M. Liu, A. Ong, F. Shao, S. Woytash, Q. Zheng and especially G. Cheang and A. Romano. Stony Brook's Fall 2010 BIO367 class and G. Cheang assisted in microsatellite data collection. For comments on the manuscript, we thank B. M. Fitzpatrick, J. S. Levinton, J. R. True, S. Spear and anonymous reviewers. Funding was provided to MCFR by the Gaige Award (American Society of Ichthyologists and Herpetologists) and by the George Williams Award (Department of Ecology and Evolution at Stony Brook). Additional funding was provided by NSF EF 0334923 to JJW.

\section{References}

Anthony CD, Venesky MD, Hickerson C-AM (2008) Ecological separation in a polymorphic terrestrial salamander. Journal of Animal Ecology, 77, 646-653.

Blankers T, Adams DC, Wiens JJ (2012) Ecological radiation with limited morphological diversification in salamanders. Journal of Evolutionary Biology, 25, 634-646.

Bloom AL (1983) Sea level and coastal morphology of the United States through the Late Wisconsin glacial maximum. In: Late-Quaternary environments of the United States, Vol. 1. The Late Pleistocene (ed. Porter SC), pp. 215-229. University of Minnesota, Minneapolis, Minnesota.

Bloom AL, Stuiver M (1963) Submergence of the Connecticut coast. Science, 139, 332-334.

Bolnick DI, Fitzpatrick BM (2007) Sympatric speciation: models and empirical evidence. Annual Review of Ecology, Evolution and Systematics, 38, 459-487.

Cavalli-Sforza LL, Edwards AWF (1967) Phylogenetic analysis: models and estimation procedures. American Journal of Human Genetics, 19, 233-257.

Connors LM, Cabe PR (2003) Isolation of dinucleotide microsatellite loci from red-backed salamander (Plethodon cinereus). Molecular Ecology Notes, 3, 131-133.

Coulon A, Fitzpatrick JW, Bowman R et al. (2008) Congruent population structure inferred from dispersal behaviour and intensive genetic surveys of the threatened Florida scrub-jay (Aphelocoma corulescens). Molecular Ecology, 17, 1685-1701.

Coyne JA (2007) Sympatric speciation. Current Biology, 17, R787-R788.

Coyne JA, Orr HA (2004) Speciation. Sinauer Associates Inc, Sunderland, Massachusetts.

Doebeli M, Dieckmann U (2000) Evolutionary branching and sympatric speciation caused by different types of ecological interactions. American Naturalist, 156, S77-S101.

Doebeli M, Dieckmann U (2003) Speciation along environmental gradients. Nature, 421, 259-264.

Earl DA, vonHoldt BM (2011) Structure Harvester: a website and program for visualizing Structure output and implementing the Evanno method. Conservation Genetics Research, 4, 359-361.

Evanno G, Regnaut S, Goudet J (2005) Detecting the number of clusters of individuals using the software Structure: a simulation study. Molecular Ecology, 14, 2611-2620.

Falush D, Stephens M, Pritchard JK (2003) Inference of population structure: extensions to linked loci and correlated allele frequencies. Genetics, 164, 1567-1587.

Felsenstein J (2004) PHYLIP (Phylogeny Inference Package). Distributed by the author, Department of Genome Sciences, University of Washington, Seattle.

Fisher-Reid MC, Wiens JJ (2011) What are the consequences of combining nuclear and mitochondrial data for phylogenetic analysis? Lessons from Plethodon salamanders and 13 other vertebrate clades. BMC Evolutionary Biology, 11, 300.

Fisher-Reid MC, Kozak KH, Wiens JJ (2012) How is the rate of climatic-niche evolution related to climatic-niche breadth? Evolution, 66, 3836-3851.

Fitzpatrick BM, Shook K, Izally R (2009) Frequency-dependent selection by wild birds promotes polymorphism in model salamanders. BMC Ecology, 9, 12.

Gavrilets S (2004) Fitness Landscapes and the Origin of Species. Princeton University Press, Princeton, New Jersey. 
Gibbs JP, Karraker NE (2006) Effects of warming conditions in eastern North American forests on red-backed salamander morphology. Conservation Biology, 20, 913-917.

Glenn TC, Schable NA (2005) Isolating microsatellite DNA loci. Methods in Enzymology, 395, 202-222.

Guillot G, Rousset F (2013) Dismantling the Mantel tests. Methods in Ecology and Evolution, 4, 336-344.

Highton R (1975) Geographic variation in genetic dominance of the color morphs of the red-backed salamander, Plethodon cinereus. Genetics, 80, 363-374.

Highton R (1977) Comparison of microgeographic variation in morphological and electrophoretic traits. Evolutionary Biology, 10, 397-436.

Hijmans RJ, Cameron SE, Parra JL, Jones PG, Jarvis A (2005) Very high resolution interpolated climate surfaces for global land areas. International Journal of Climatology, 25, 1965-1978.

Hubisz MJ, Falush D, Stephens M, Pritchard JK (2009) Inferring weak population structure with the assistance of sample group information. Molecular Ecology Resources, 9, 1322-1332.

Ingram T (2011) Speciation along a depth gradient in a marine adaptive radiation. Proceedings of the Royal Society of London, Series B, 278, 613-618.

Jackson DA (1993) Stopping rules in principal components analysis: a comparison of heuristical and statistical approaches. Ecology, 74, 2204-2214.

Jockusch EL (1997) Geographic variation and phenotypic plasticity of number of trunk vertebrae in slender salamanders, Batrachoseps (Caudata: Plethodontidae). Evolution, 51, 19661982.

Jordan MA, Morris DA, Gibson SE (2009) The influence of historical landscape change on genetic variation and population structure of a terrestrial salamander (Plethodon cinereus). Conservation Genetics, 10, 1647-1658.

Kozak KH, Wiens JJ (2007) Climatic zonation drives latitudinal variation in speciation mechanisms. Proceedings of the Royal Society of London, Series B, 274, 2995-3003.

Kozak KH, Wiens JJ (2010) Accelerated rates of climatic-niche evolution underlie rapid species diversification. Ecology Letters, 13, 1378-1389.

Kozak KH, Mendyk RW, Wiens JJ (2009) Can parallel diversification occur in sympatry? Repeated patterns of body-size evolution in co-existing clades of North American salamanders. Evolution, 63, 1769-1784.

Legendre P, Legendre L (1998) Numerical Ecology, 2nd English edn. Elsevier Science B.V, Amsterdam.

Lewis RS, Stone JR (1991) Late Quaternary stratigraphy and depositional history of the Long Island Sound basin: Connecticut and New York. Journal of Coastal Research SI11, 1-23.

Lotter F, Scott NJ Jr (1977) Correlation between climate and distribution of the color morphs of the salamander Plethodon cinereus. Copeia, 1977, 681-690.

Maddison WP, Maddison DR (2011) Mesquite: A modular system for evolutionary analysis. Version $2.75 \mathrm{http}$ ://mesquiteproject.org.

Mantel NA (1967) The detection of disease clustering and a generalized regression approach. Cancer Research, 27, 209220.

Moreno G (1989) Behavioral and physiological differentiation between the color morphs of the salamander, Plethodon cinereus. Journal of Herpetology, 23, 335-341.
Moritz C, Patton JL, Schneider CJ, Smith TB (2000) Diversification of rainforest faunas: an integrated molecular approach. Annual Review of Ecology and Systematics, 31, 533-563.

Noël S, Lapointe F-J (2010) Urban conservation genetics: study of a terrestrial salamander in the city. Biological Conservation, 143, 2823-2831.

Nosil P (2008) Speciation with gene flow could be common. Molecular Ecology, 17, 2103-2106.

Petranka JW (1998) Salamanders of the United States and Canada. Smithsonian Institution Press, Washington, District of Columbia.

Petruzzi EE, Niewiarowski PH, Moore FB-G (2006) The role of thermal niche selection in maintenance of a colour polymorphism in redback salamanders (Plethodon cinereus). Frontiers in Zoology, 3, 10.

Pritchard JK, Stephens M, Donnelly P (2000) Inference of population structure using multilocus genotype data. Genetics, 155, 945-959.

Pritchard JK, Wen X, Falush D (2010) Documentation for structure software: Version 2.3.

Raymond M, Rousset F (1995) Genepop (version 1.2): population genetics software for exact tests and ecumenicism. Journal of Heredity, 86, 248-249.

Rice WR (1989) Analyzing tables of statistical tests. Evolution, 43, 223-225.

Rosenberg MS, Anderson CD (2011) PASSaGE: pattern analysis, spatial statistics, and geographic exegesis. Version 2. Methods in Ecology and Evolution, 2, 229-232.

Rousset F (2008) Genepop'007: a complete reimplementation of the Genpop software for Windows and Linux. Molecular Ecology Resources, 8, 103-106.

Seehausen O, Terai Y, Magalhaes IS et al. (2008) Speciation through sensory drive in cichlid fish. Nature, 455, 620-626.

Smouse PE, Long JC, Sokal RR (1986) Multiple regression and correlation extensions of the Mantel Test of matrix correspondence. Systematic Zoology, 35, 627-632.

Sobel JM, Chen GF, Watt LR, Schemske DW (2010) The biology of speciation. Evolution, 64, 295-315.

Stamatakis A (2006) RAxML-VI-HPC: maximum likelihoodbased phylogenetic analyses with thousands of taxa and mixed models. Bioinformatics, 22, 2688-2690.

Stone JR, Schafer JP, London EH et al. (2005) Quaternary geologic map of Connecticut and Long Island Sound basin. United States Geological Survey Scientific Investigations Map 2784.

Takezaki N, Nei M (1996) Genetic distances and reconstruction of phylogenetic trees from microsatellite DNA. Genetics, 144, 389-399.

Tilley SG, Lundrigan BL, Brower LP (1982) Erythrism and mimicry in the salamander Plethodon cinereus. Herpetologica, 38, 409-417.

Wake DB (1966) Comparative osteology and evolution of the lungless salamanders, family Plethodontidae. Memoirs Southern California Academy of Sciences, 4, 1-111.

Weir BS, Cockerham CC (1984) Estimating F-statistics for the analysis of population structure. Evolution, 38, 1358-1370.

Wiens JJ, Engstrom TN, Chippindale PT (2006) Rapid diversification, incomplete isolation, and the 'speciation clock' in North American salamanders (genus Plethodon): testing the hybrid swarm hypothesis of rapid radiation. Evolution, 60, 2585-2603. 
Williams EE, Highton R, Cooper DM (1968) Breakdown of polymorphism of the red-backed salamander on Long Island. Evolution, 22, 76-86.

This paper is part of M.C.F.R.'s Ph.D. dissertation on speciation in salamanders. M.C.F.R. collected all microclimatic data, microsatellite data (with C.A.K.), conducted all analyses and wrote the manuscript (with J.J.W.). T.N.E. and J.J.W. conceived the project. J.J.W., T.N.E. and P.R.S. collected specimens. T.N.E. and C.A.K. collected mtDNA data. C.A.K. developed the microsatellite loci. All authors participated in fieldwork.

\section{Supporting information}

Additional supporting information may be found in the online version of this article.

Data S1. Microsatellite and heterozygosity data.

Data S2. Macroclimatic data.

Data S3. Microclimatic data.

Data S4. Morphological data.

Data S5. Data from Delmarva Peninsula.
Text S1. Methodological details.

Text S2. Analytical details.

Table S1. Locality sampling information.

Table S2. Individual specimen information, GenBank accession numbers.

Table S3. Primer information, PCR protocols.

Table S4. Macroclimate PCA results.

Table S5. STRUCTURE results.

Table S6. Microclimate statistical analyses.

Table S7. Morphology PCA results.

Table S8. $F_{\mathrm{ST}}$, geographic distance for select populations.

Table S9. Mantel test results.

Fig. S1. Trees of mtDNA haplotypes.

Fig. S2. Map of geographic midpoints of salamander population categories on LI.

Fig. S3. Ancestral reconstruction using locality morph frequency as a continuous trait.

Fig. S4. PC1 vs. PC2 for morphological PCAs. 